\title{
Semi-classical dynamical triangulations
}

\author{
J. Ambjørn ${ }^{\mathrm{a}, \mathrm{b}, *}$, T.G. Budd ${ }^{\mathrm{a}}$ \\ a The Niels Bohr Institute, Copenhagen University, Blegdamsvej 17, DK-2100 Copenhagen Ø, Denmark \\ ${ }^{\mathrm{b}}$ Institute for Mathematics, Astrophysics and Particle Physics (IMAPP), Radbaud University Nijmegen, Heyendaalseweg 135, 6525 AJ, Nijmegen, The Netherlands
}

\section{A R T I C L E I N F O}

\section{Article history:}

Received 26 September 2012

Accepted 3 October 2012

Available online 8 October 2012

Editor: L. Alvarez-Gaumé

\section{Keywords:}

Quantum gravity

Lower dimensional models

Lattice models

\begin{abstract}
A B S T R A C T
For non-critical string theory the partition function reduces to an integral over moduli space after integrating over matter fields. The moduli integrand is known analytically for genus one surfaces. The formalism of dynamical triangulations provides us with a regularization of non-critical string theory and we show that even for very small triangulations it reproduces very well the continuum integrand when the central charge $c$ of the matter fields is large negative, thus providing a striking example of how the quantum fluctuations of geometry disappear when $c \rightarrow-\infty$.
\end{abstract}

(c) 2012 Elsevier B.V. All rights reserved.

\section{Introduction}

Non-critical string theory (or equivalently 2d Euclidean quantum gravity coupled to conformal field theories) has allowed us to study non-perturbative aspects of string theory and quantum gravity. Certain aspects of the theory can be solved by continuum methods and certain aspects have been solved using combinatorial methods (matrix models and dynamical triangulations (DT)). Originally dynamical triangulations were considered as a lattice regularization of path integral over two dimensional world sheet geometries [1-3], but the versatility of the matrix models allowed for many other interesting interpretations, which became increasingly detached from the original idea of DT as a lattice regularization of a $2 \mathrm{~d}$ quantum world.

A typical dynamical triangulation represents a (piecewise linear) geometry which appears in the path integral over $2 \mathrm{~d}$ geometries. As such it does not represent anything like a physical measurable geometry, in the same way as a random path in the path integral representation of the particle is not physical and cannot be measured. However, it has interesting fractal properties, precise as the random path for the particle with probability 1 has Hausdorff dimension 2. The "random geometries" in the path integral (represented in DT by dynamical triangulations) have Hausdorff

\footnotetext{
* Corresponding author at: The Niels Bohr Institute, Copenhagen University, Blegdamsvej 17, DK-2100 Copenhagen Ø, Denmark.

E-mail addresses: ambjorn@nbi.dk (J. Ambjørn), budd@nbi.dk (T.G. Budd).
}

dimension

$$
d_{h}=2 \frac{\sqrt{49-c}+\sqrt{25-c}}{\sqrt{25-c}+\sqrt{1-c}} .
$$

Contrary to the particle case, this is an intrinsic fractal dimension, not a fractal dimension in target space. Also, it depends on the central charge of conformal field theory coupled to $2 \mathrm{~d}$ gravity. This result was first obtained for $c=0$ in [4] using the DT formalism, and later, again using the DT formalism, from a different perspective in [5,6]. The general formula was derived in [7] using Liouville theory.

These results indicate that for a given central charge $c$ there exists a measure defined on the set of continuous geometries. However, no precise mathematical definition exists yet, contrary to the situation for continuous paths (the Wiener measure). In critical string theory this problem is circumvented by using the conformal invariance of the theory. In this way most of the integration over geometries can be factored out, and we are left only with a finite dimensional integration over the moduli parameters (and a nontrivial integrand for higher genus surfaces). In non-critical string theory the situation is more complicated due to the zero mode of the Liouville field, except in the genus one case. In this case one obtains just the standard result from critical string theory [8]. Thus, if we consider the situation where we fix the world sheet area to be $A$ we obtain

$$
Z^{(h=1)}(A) \sim A^{-1} \int_{\mathcal{M}} \frac{d^{2} \tau}{\tau_{2}^{2}} F(\tau)^{c-1},
$$




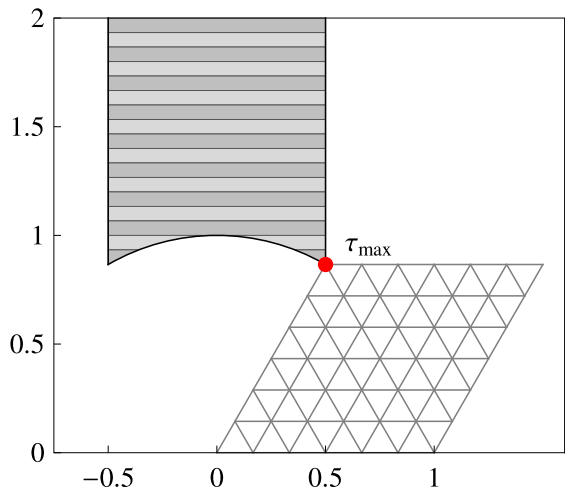

Fig. 1. The fundamental domain $\mathcal{M}$ in the upper-half complex plane. Its coloring illustrates the choice of bins used in Fig. 3. The maximum of $F(\tau)^{1-c}$ at $\tau_{\max }=\mathrm{e}^{i \pi / 3}$ corresponds to the equilateral torus, i.e. the quotient of the plane by the hexagonal lattice.

where $\tau=\tau_{1}+i \tau_{2}$ is the moduli parameter, $\mathcal{M}$ denotes the fundamental domain of $\tau$ in the upper complex plane ${ }^{1}$ and

$$
F(\tau)=\tau_{2}^{-1 / 2}|\eta(\tau)|^{-2}=\tau_{2}^{-1 / 2} \mathrm{e}^{\pi \tau_{2} / 6} \prod_{n=1}^{\infty}\left|1-\mathrm{e}^{2 \pi i n \tau}\right|^{-2} .
$$

The integral in (2) diverges for $c>1$, which tells us that there is a $c=1$ barrier beyond which the formulas make little sense. When $c$ decreases geometries with large $\tau_{2}$ become more and more suppressed. In fact the maximum of $F(\tau)^{1-c}$, located at $\tau_{\max }=\mathrm{e}^{i \pi / 3}$ in the fundamental domain, will dominate the integral for $c \rightarrow-\infty$ (see Fig. 1).

By assigning moduli parameters to triangulations of the torus one can study how the formalism of DT reproduces the integrand in (2). The possibility to assign a moduli parameter to a toroidal triangulation was first realized in [9], where the first study of the DT-realization of Eq. (2) was performed. Using the formalism of simplicial cohomology this assignment can be done in a natural and relatively simple way [10], and one can even explicitly construct the harmonic map from the geometry defined by the triangulation to the parallelogram defined by the moduli parameter. Stated briefly, given two independent cycles on a torus, $\gamma_{1}$ and $\gamma_{2}$, one can find dual harmonic one-forms $\alpha_{1}$ and $\alpha_{2}$ such that

$$
\int_{\gamma_{i}} \alpha^{j}=\delta_{i}^{j}
$$

Then a moduli parameter of the torus is given by

$$
\tau=-\frac{\left\langle\alpha^{1} \mid \alpha^{2}\right\rangle}{\left\langle\alpha^{2} \mid \alpha^{2}\right\rangle}+i \sqrt{\frac{\left\langle\alpha^{1} \mid \alpha^{1}\right\rangle}{\left\langle\alpha^{2} \mid \alpha^{2}\right\rangle}-\left(\frac{\left\langle\alpha^{1} \mid \alpha^{2}\right\rangle}{\left\langle\alpha^{2} \mid \alpha^{2}\right\rangle}\right)^{2}},
$$

where $\langle\cdot, \cdot\rangle$ refers to the standard inner product on one-forms. This $\tau$ is not necessarily in the fundamental domain $\mathcal{M}$, but is uniquely associated with a point there by a modular transformation. The concepts of cycles, one-forms and harmonic one-forms have a natural formulation on complexes, the de Rham cohomology being replaced by simplicial cohomology. In the case of DT, where the triangles are equilateral, all formulas become very simple. On toroidal triangulations we can first find cycles (non-contractible link-loops), then the dual one-forms (harmonic in the simplicial context), the

\footnotetext{
${ }^{1}$ A convenient choice for the fundamental domain $\mathcal{M}$ is (see Fig. 1):

$\tau \in \mathcal{M}$ if $\begin{cases}\tau_{2}>0, & -\frac{1}{2}<\tau_{1}<0 \text { and }|\tau|>1 \\ \tau_{2}>0, & 0 \leqslant \tau_{1} \leqslant \frac{1}{2} \text { and }|\tau| \geqslant 1\end{cases}$
}

corresponding $\tau$ and finally the modular transformation which maps it to the fundamental domain. We refer to [10] for details.

In $[10,11]$ this formalism was used to study $2 d$ Euclidean quantum gravity for the matter central charges $c=0$ and $c=-2$. These values were chosen since they allowed for large computer generated triangulations with the correct weights. Large triangulations were necessary in order to properly include the singular geometries with large $\tau_{2}$. In this Letter we extend the study to large negative $c$. For such $c$ we expect geometries with large $\tau_{2}$ to be suppressed and therefore smaller triangulations should suffice. As we will explain below, relatively small triangulations can be computer generated with the correct weight for any $c$.

\section{Large negative valued central charge}

Consider the partition function for the bosonic string in $d$ dimensions:

$Z^{(h)}=\int \mathcal{D}[g] \int \mathcal{D}_{g} X_{\mu} \mathrm{e}^{-S(X, g)}$,

where the integration is over worldsheet geometries $[g]$ of genus $h$ and Gaussian matter fields $X_{\mu}$. The $d$ Gaussian fields can be integrated out and we obtain

$\int \mathcal{D}_{g} X_{\mu} \mathrm{e}^{-S(X, g)} \sim\left(\operatorname{det}\left(-\Delta_{g}^{\prime}\right)\right)^{-d / 2}$.

Here $\Delta_{g}^{\prime}$ denotes the Laplace-Beltrami operator on the background geometry defined by a metric $g_{a b}$ and the prime signifies that the zero mode has been removed when calculating the determinant of $\Delta_{g}$. If we consider $d$ as a formal parameter we can continue this expression to negative $d$. For positive integer $d$ we can identify $d$ with the central charge $c$ of a conformal field theory coupled to the $2 \mathrm{~d}$ world sheet geometry and formally we can do the same for any real $d$. The DT formalism tells us that we should represent the regularized partition function (7) as

$Z^{(h=1)}(N)=\sum_{T_{N}} \frac{1}{C_{T_{N}}}\left(\operatorname{det}\left(-\Delta_{T_{N}}^{\prime}\right)\right)^{-d / 2}$,

where the summation is over all triangulations of the torus with $N$ triangles. $C_{T}$ is the symmetry factor of the triangulation $T$, i.e. the order of the automorphism group of the graph $T$. Finally $\Delta_{T}$ denotes the (discretely defined) Laplacian on the DT-surface, which we take to be the usual graph Laplacian of the $\phi^{3}$ graph dual to the triangulation. The continuum area $A$ in formula (2) is related to $N$ by $A=N a^{2} \sqrt{3} / 2$, where $a$ denotes the length of a link in the triangulation constructed from equilateral triangles. The continuum limit of DT is obtained by taking $a \rightarrow 0$ while keeping $A$ fixed. Thus we only expect the DT result to be exact (i.e. to agree with the continuum expression) for $N \rightarrow \infty$.

As long as $N$ is not much larger than a few hundred triangles standard Monte Carlo simulations can be used to generate an ensemble of triangulations $\left\{T_{i}(N)\right\}$ with the correct weight dictated by the partition function (9). We refer to Appendix A for details on the updating algorithm used in the simulations. For each triangulation $T_{i}(N)$ we can now extract the moduli parameter by the technique mentioned above and described in detail in [10]. As mentioned before one can obtain an explicit harmonic map from the triangulation to the parallelogram in the Euclidean plane defined by the moduli parameters. Fig. 2 shows examples of such maps for typical triangulations appearing in the partition functions for various values of the central charge. These examples indicate that typical triangulations become more and more regular, i.e. closer to the hexagonal lattice in Fig. 1, when $c$ becomes more negative. From the measurements we construct a probability 
$c=-5$

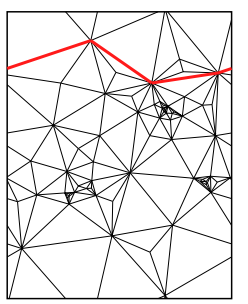

$c=-10$

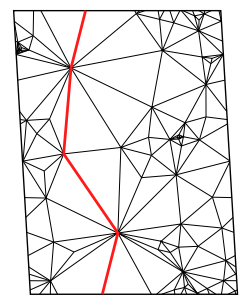

$c=-20$

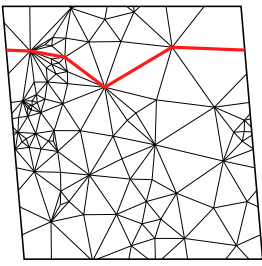

$c=-40$ $c=-80$

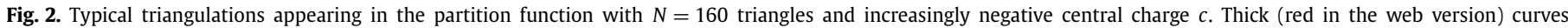
correspond to the shortest non-contractible loops studied in Section 3.
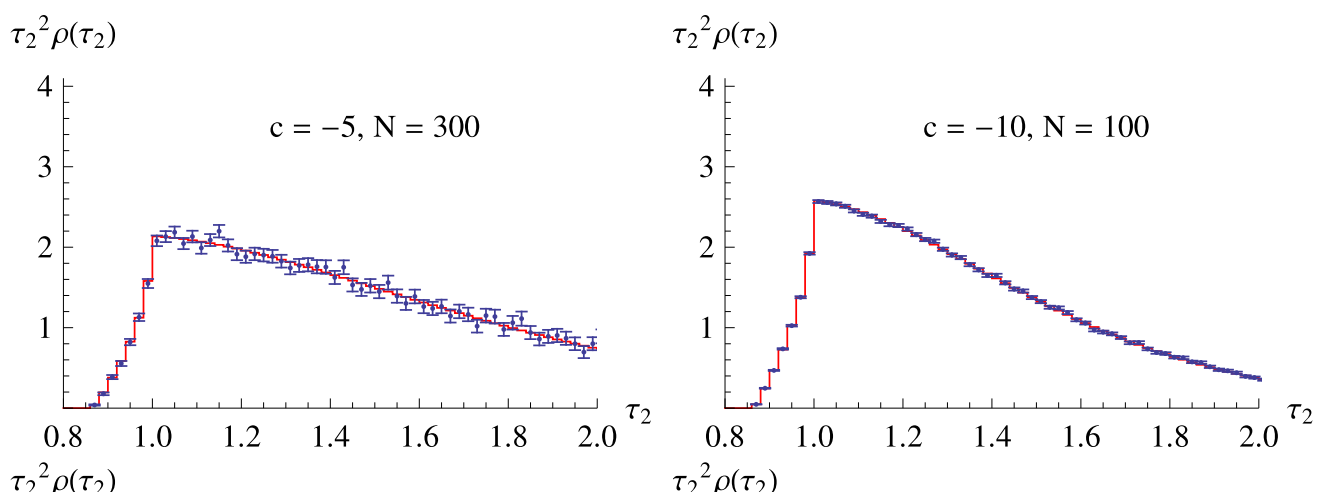

$\tau_{2}^{2} \rho\left(\tau_{2}\right)$

$\tau_{2}^{2} \rho\left(\tau_{2}\right)$
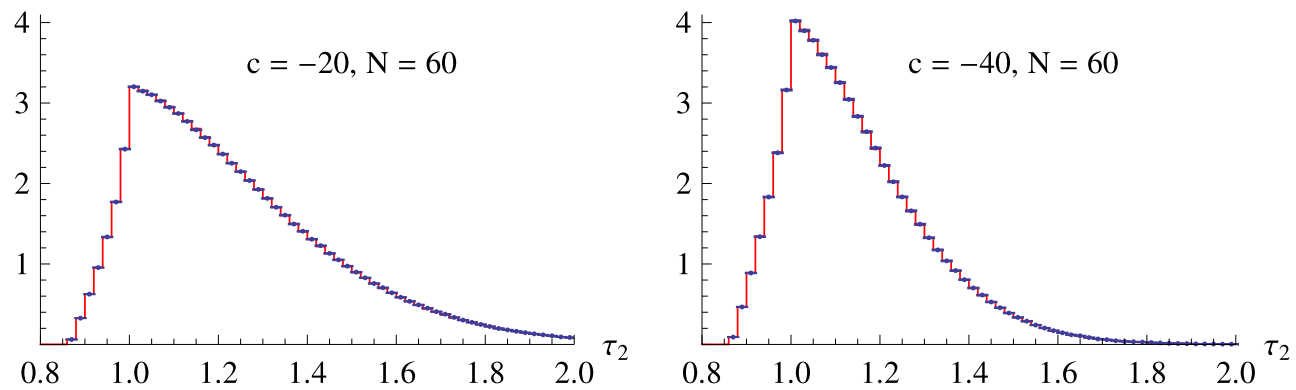

Fig. 3. The $\tau_{2}$ distributions $\tau_{2}^{2} \rho\left(\tau_{2} ; N\right)$ compared to the theoretical distributions. The data points and the theoretical curves are produced as described in the main text.

distribution in the fundamental domain $\mathcal{M}$ which we can compare with the continuum integrand $F(\tau)^{c-1} / \tau_{2}^{2}$ in (2). The errors one encounters are twofold. There will be statistical errors which diminish with the size of the computer generated ensemble $\left\{T_{N}\right\}$ in the standard way, and there will be systematic errors associated with the use a finite number $N$ of triangles.

Our results are shown in Fig. 3. The figures are constructed in the following way. The fundamental domain $\mathcal{M}$ is partitioned into bins according to the value of $\tau_{2}$ with bin size $\Delta \tau_{2}=0.025$, as illustrated in Fig. 1 (however notice that there we have displayed bins with larger bin size for visualization purposes). We have chosen to disregard the value of $\tau_{1}$ since the theoretical integrand $F(\tau)^{c-1} / \tau_{2}^{2}$ varies little with $\tau_{1}$. We denote by $\rho\left(\tau_{2}\right) \Delta \tau_{2}$ the probability of observing a $\tau\left(T_{i}(N)\right)$ associated with a triangulation $T_{i}(N)$ sitting in the bin $\tau_{2}-\frac{1}{2} \Delta \tau_{2} \leqslant \tau_{2}\left(T_{i}(N)\right)<\tau_{2}+\frac{1}{2} \Delta \tau_{2}$. We compare $\rho\left(\tau_{2}\right)$ to the theoretical distribution $\rho_{\text {theory }}\left(\tau_{2}\right)$ obtained by integrating $F(\tau)^{c-1} / \tau_{2}^{2}$ over the corresponding bin. The measured distributions for various values of the central charge are depicted in Fig. 3 by points with error-bars (which are hardly visible for $c \leqslant-10$ ) and the solid lines correspond to the theoretical distributions.

From Fig. 3 it is seen that $\tau_{2}^{2} \rho\left(\tau_{2}, N\right)$ is more and more concentrated at the smallest possible $\tau_{2}$ values when $c$ becomes increasingly negative, as expected from the theoretical distribution. Also, in order to obtain a reasonable accuracy we have to take
Table 1

The fit $c_{f i t}$ to the integrand in (2) from data.

\begin{tabular}{lllll}
\hline$c$ & -5 & -10 & -20 & -40 \\
\hline$c_{\text {fit }}$ & $-5.01 \pm 0.1$ & $-9.94 \pm 0.04$ & $-19.9 \pm 0.1$ & $-39.88 \pm 0.05$ \\
\hline
\end{tabular}

$N=300$ for $c=-5$, while for $c=-40$ one obtains good results already with $N=60$. The need for large $N$ the closer $c$ is to barrier $c=1$ reflects that the distribution $\rho\left(\tau_{2}\right)$ becomes more flat when $c$ increases. Thus both $\left\langle\tau_{2}\right\rangle$ and $\left\langle\tau_{2}^{2}\right\rangle-\left\langle\tau_{2}\right\rangle^{2}$ increase. However, the large $\tau_{2}$ region is not well reproduced by triangulations with small $N$, the reason being that loops take integer length and, loosely speaking, $\tau_{2}$ is related to the ratio between the two independent shortest non-contractible loops. When we get closer to $c=1$, the effective area of the fundamental domain $\mathcal{M}$ that has to be covered by the simulations becomes larger. Because of this and the need for larger triangulations, more and more computertime is needed. This trend is reflected well in the figures and the message is: the semi-classical region $c \rightarrow-\infty$ is easy!

As further evidence of the precision with which the simplicial cohomology is able to reproduce the continuum results in the range of central charges that we have considered here, we have fit the data in Fig. 3 to the theoretical distribution with the central charge $c$ in formula (2) as a free parameter. The result is listed in Table 1. 

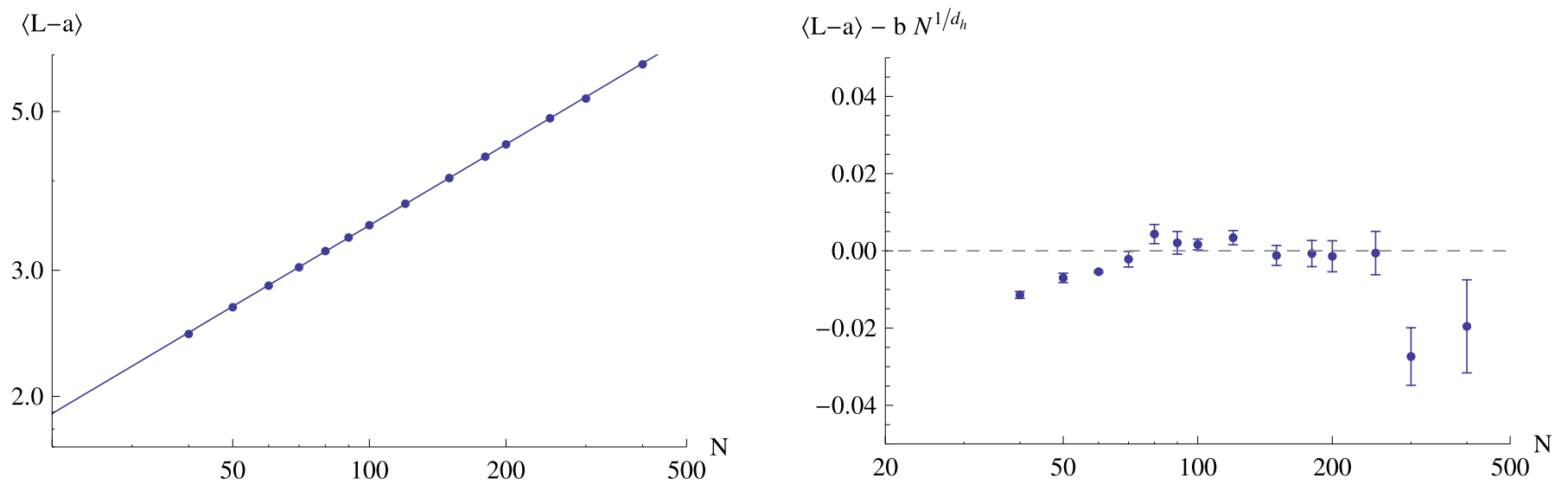

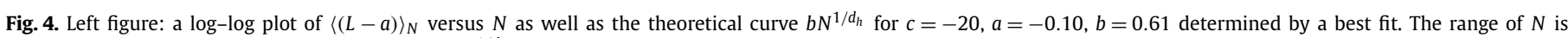
30-400. Right figure: the difference $\langle(L-a)\rangle_{N}-b N^{1 / d_{h}}$.

\section{The Hausdorff dimension}

One of the most remarkable formulas in 2d Euclidean quantum gravity is (1). Why do we need to verify it? There have been a number of competing formulas [13]. Although it is mostly believed today that these formulas describe critical properties of the matter theories coupled to $2 \mathrm{~d}$ gravity rather than the fractal structure of a typical geometry which appears in the path integral [14], the question is not completely settled. In addition, the derivation of (1) is based on a certain number of assumptions related to diffusion on fluctuating geometries, assumptions which are not necessarily valid.

In $[10,11]$ formula (1) was verified with great numerical accuracy for $c=0$ and $c=-2$. Here we check the formula for more negative $c$. As noted in $[10,11]$ the torus offers a nice opportunity for a numerical check of the formula which is independent of the original numerical check [12] performed by simply measuring the area $A(r)$ enclosed in disks of geodesic radius $r$ :

$\langle A(r)| \sim r^{d_{h}}, \quad r \ll A^{1 / d_{h}}$,

where $A$ is the fixed area of the $2 d$ geometry, as in formula (2). On the torus one can use that in a given geometry a shortest non-contractible loop must be a geodesic curve. On average a non-contractible loop will have a length $\langle L\rangle_{A} \sim A^{1 / 2}$. However, if the geodesic distances scale anomalously, as is the case if $d_{h} \neq 2$ in (10), we expect instead

$\langle L\rangle_{A} \sim A^{1 / d_{h}}$.

In DT we can perform such measurements: we define curves as link-paths, and a geodesic curve between two vertices as a shortest link-path. Let now $\left\{T_{i}(N)\right\}$ denote the DT ensemble of triangulations with $N$ triangles generated with the correct weight. Fixed $N$ corresponds to fixed continuum area $A$ in this context. For each triangulation we determine a shortest non-contractible loop ${ }^{2}$ and in this way we can calculate the average $\langle L\rangle_{N}$. We do this for as large range of $N$ as possible and determine $d_{h}$ from a fit to (11). We have chosen $c=-20$ and $N$ in the range from 30 to 400 . A best three parameter fit $^{3}$

\footnotetext{
2 The practical algorithm used to find the shortest non-contractible loop uses in a nice way simplicial cohomology. We refer to [10] for details. See also Fig. 2 for examples of shortest non-contractible loops.

3 The parameter $a$ in (12) is not present in (11), but is quite natural. It reflects the fact that $L$ in DT is an integer, contrary to the $L$ in the continuum formula. It
}

$\langle(L-a)\rangle_{N}=b N^{1 / d} \rightarrow d=2.76 \pm 0.07$,

which should be compared to the theoretical value $d_{h} \approx 2.66$. In view of the small values of $N$ used we consider this result as quite satisfactory and a confirmation of formula (1). The left figure in Fig. 4 shows the measured $\langle(L-a)\rangle_{N}$ together with the theoretical curve $b N^{1 / d_{h}}$ (where $a, b$ is determined by a best fit). The right figure in Fig. 4 displays the error in the fit. It is clear that $N$ is too small to produce a perfect fit. If one leaves out the three smallest values of $N$ one obtains a $\chi^{2}$ value of order 2 , which is good.

\section{Discussion}

Matrix models have provided convincing evidence that the formalism of dynamical triangulations provides a regularization of non-critical string theory or equivalently $2 \mathrm{~d}$ Euclidean quantum gravity coupled to matter with central charge $c \leqslant 1$. However, quantum geometric aspects of $2 \mathrm{~d}$ Euclidean quantum gravity are not easily analyzed using matrix models. The reason is that when using matrix models one integrates over all geometries. The matrix model thus provides us with the full partition function of matter and gravity and to obtain more detailed information one has to insert certain "punctures" and calculate their expectation values. These operators also mix matter and geometry and to disentangle geometry and matter is not easy and becomes increasing complicated in the limit $c \rightarrow-\infty$, a limit where one naively would expect the quantum aspects of geometry to decouple. Formula (1) can be read as an explicit, quantitative statement about this decoupling: $d_{h} \rightarrow 2$ for $c \rightarrow-\infty$. However, it is not derived using matrix models but quantum Liouville theory.

One would expect that the original interpretation of DT, where the triangulations are viewed as a lattice regularization of the path integral over geometries, should allow us to observe the increased semi-classical nature of geometry in detail. The purpose of this Letter was to provide evidence for this. For the torus we have shown that the formalism of DT indeed reproduces the integrand in (2), not only the partition function. Further, the more negative the value of $c$, the better is the continuum expression reproduced even for small triangulations. While conformal invariance of the continuum theory blurs somewhat the concept of a regular geometry, the possibility to use the moduli parameter of the torus has

can be viewed as a finite size correction, using the terminology of finite size scaling in the theory of critical phenomena. 


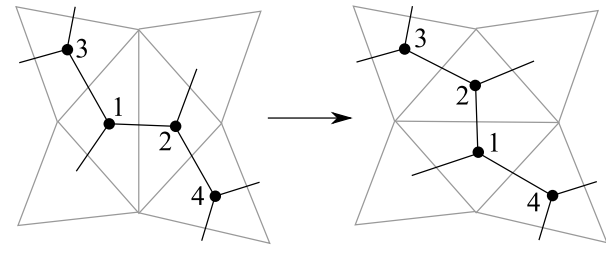

Fig. 5. Flip move.

allowed us to address the problem in a conformally invariant way, and in the $c \rightarrow-\infty$ limit we clearly see the dominance of the "regular" triangulations, i.e. triangulations of the torus which have small $\tau_{2}$ values.

Finally, using the concept of a shortest non-contractible loop on the torus, we have been able to test and (to some degree) verify formula (1).

\section{Acknowledgements}

The authors acknowledge support from the ERC-Advance grant 291092, "Exploring the Quantum Universe" (EQU). J.A. acknowledges support of FNU, the Free Danish Research Council, from the grant "Quantum gravity and the role of black holes". Part of this research was carried out at Utrecht University, The Netherlands.

\section{Appendix A. Monte Carlo simulations}

In order to generate random triangulations according to the distribution (9) we use standard Monte Carlo methods. We start with some initial triangulation with the desired number $N$ of triangles. Then we apply random updates to the triangulation via so-called flip moves, as shown in Fig. 5. To obtain the correct Boltzmann distribution one has to implement an acceptance probability that is related to the ratio of Boltzmann factors before and after the flip move, i.e.

$p=\left[\frac{\operatorname{det}^{\prime}(-\Delta-\delta \Delta)}{\operatorname{det}^{\prime}(-\Delta)}\right]^{-d / 2}=\left[\operatorname{det}\left(I+\Delta^{-1} \delta \Delta\right)\right]^{-d / 2}$.

Here $\delta \Delta$ is the change under the flip move of the Laplacian on the dual graph of the triangulation, and $\Delta^{-1}$ is the pseudo-inverse of $\Delta$. The matrix $\delta \Delta$ has only eight non-vanishing entries and, adopting the labeling in Fig. 5, is given by

$\delta \Delta=\left(\begin{array}{ccccc}0 & 0 & -1 & 1 & \ldots \\ 0 & 0 & 1 & -1 & \ldots \\ -1 & 1 & 0 & 0 & \ldots \\ 1 & -1 & 0 & 0 & \ldots \\ \vdots & \vdots & \vdots & \vdots & \ddots\end{array}\right)$.

Therefore (13) reduces to a determinant of a four by four matrix $p=\left|\begin{array}{cccc}1-\psi_{1} & \psi_{1} & -\phi_{1} & \phi_{1} \\ \psi_{1} & 1-\psi_{1} & \phi_{1} & -\phi_{1} \\ -\psi_{3} & \psi_{3} & 1-\phi_{3} & \phi_{3} \\ \psi_{3} & -\psi_{3} & \phi_{3} & 1-\phi_{3}\end{array}\right|^{-d / 2}$,

where the vectors $\phi$ and $\psi$ are the solutions to the linear equations

$\Delta \phi=\left(\begin{array}{c}1 \\ -1 \\ 0 \\ 0 \\ \vdots\end{array}\right), \quad \Delta \psi=\left(\begin{array}{c}0 \\ 0 \\ 1 \\ -1 \\ \vdots\end{array}\right)$,
$\sum_{i} \phi_{i}=\sum_{i} \psi_{i}=0$.

Solving these equations using numerical linear algebra techniques is the most time-consuming step of the update move. It limits the simulations to triangulations of up to several hundred triangles.

\section{References}

[1] F. David, Nucl. Phys. B 257 (1985) 45; A. Billoire, F. David, Phys. Lett. B 168 (1986) 279.

[2] V.A. Kazakov, A.A. Migdal, I.K. Kostov, Phys. Lett. B 157 (1985) 295; D.V. Boulatov, V.A. Kazakov, I.K. Kostov, A.A. Migdal, Nucl. Phys. B 275 (1986) 641.

[3] J. Ambjorn, B. Durhuus, J. Fröhlich, Nucl. Phys. B 257 (1985) 433; J. Ambjorn, B. Durhuus, J. Fröhlich, P. Orland, Nucl. Phys. B 270 (1986) 457.

[4] N. Kawamoto, V.A. Kazakov, Y. Saeki, Y. Watabiki, Phys. Rev. Lett. 68 (1992) 2113.

[5] H. Kawai, N. Kawamoto, T. Mogami, Y. Watabiki, Phys. Lett. B 306 (1993) 19, arXiv:hep-th/9302133:

J. Ambjorn, Y. Watabiki, Nucl. Phys. B 445 (1995) 129, arXiv:hep-th/9501049.

[6] J. Ambjorn, J. Jurkiewicz, Y. Watabiki, Nucl. Phys. B 454 (1995) 313, arXiv:heplat/9507014.

[7] Y. Watabiki, Prog. Theor. Phys. Suppl. 114 (1993) 1.

[8] A. Gupta, S.P. Trivedi, M.B. Wise, Nucl. Phys. B 340 (1990) 475; M. Bershadsky, I.R. Klebanov, Phys. Rev. Lett. 65 (1990) 3088.

[9] H. Kawai, N. Tsuda, T. Yukawa, Phys. Lett. B 351 (1995) 162, arXiv:hep-th/ 9503052;

H. Kawai, N. Tsuda, T. Yukawa, Nucl. Phys. Proc. Suppl. 47 (1996) 653, arXiv:hep-lat/9512014;

H. Kawai, N. Tsuda, T. Yukawa, Nucl. Phys. Proc. Suppl. 53 (1997) 777, arXiv:hep-lat/9609002.

[10] J. Ambjorn, J. Barkley, T.G. Budd, Nucl. Phys. B 858 (2012) 267, arXiv:1110.4649 [hep-th].

[11] J. Ambjorn, J. Barkley, T. Budd, R. Loll, Phys. Lett. B 706 (2011) 86, arXiv:1110.3998 [hep-th].

[12] J. Ambjorn, K.N. Anagnostopoulos, T. Ichihara, L. Jensen, N. Kawamoto, Y. Watabiki, K. Yotsuji, Phys. Lett. B 397 (1997) 177, arXiv:hep-lat/9611032;

J. Ambjorn, K.N. Anagnostopoulos, T. Ichihara, L. Jensen, N. Kawamoto, Y. Watabiki, K. Yotsuji, Nucl. Phys. B 511 (1998) 673, arXiv:hep-lat/9706009;

J. Ambjorn, K.N. Anagnostopoulos, T. Ichihara, L. Jensen, N. Kawamoto, Y. Watabiki, K. Yotsuji, Nucl. Phys. Proc. Suppl. 63 (1998) 748, arXiv:heplat/9709063.

[13] J. Distler, Z. Hlousek, H. Kawai, Int. J. Mod. Phys. A 5 (1990) 1093; N. Ishibashi, H. Kawai, Phys. Lett. B 322 (1994) 67, arXiv:hep-th/9312047.

[14] J. Ambjorn, C. Kristjansen, Y. Watabiki, Nucl. Phys. B 504 (1997) 555, arXiv:hepth/9705202. 